University of Nebraska - Lincoln

DigitalCommons@University of Nebraska - Lincoln

Biological Systems Engineering: Papers and

Publications

Biological Systems Engineering

2014

\title{
In vitro development of preimplantation porcine embryos using alginate hydrogels as a three-dimensional extracellular matrix
}

\author{
Catherine Sargus-Patino \\ University of Nebraska-Lincoln, csargus@huskers.unl.edu \\ Elane C. Wright \\ USDA-ARS US Meat Animal Research Center, Clay Center, NE \\ Sarah A. Plautz \\ University of Nebraska-Lincoln, sarah.plautz@unl.edu \\ Jeremy R. Miles \\ USDA-ARS US Meat Animal Research Center, Clay Center, NE, jeremy.miles@usda.gov \\ Jeff L. Vallet \\ USDA-ARS US Meat Animal Research Center, Clay Center, NE
}

See next page for additional authors

Follow this and additional works at: https://digitalcommons.unl.edu/biosysengfacpub

Part of the Bioresource and Agricultural Engineering Commons, Environmental Engineering Commons, and the Other Civil and Environmental Engineering Commons

Sargus-Patino, Catherine; Wright, Elane C.; Plautz, Sarah A.; Miles, Jeremy R.; Vallet, Jeff L.; and Pannier, Angela $\mathrm{K}$., "In vitro development of preimplantation porcine embryos using alginate hydrogels as a threedimensional extracellular matrix" (2014). Biological Systems Engineering: Papers and Publications. 427. https://digitalcommons.unl.edu/biosysengfacpub/427

This Article is brought to you for free and open access by the Biological Systems Engineering at DigitalCommons@University of Nebraska - Lincoln. It has been accepted for inclusion in Biological Systems Engineering: Papers and Publications by an authorized administrator of DigitalCommons@University of Nebraska Lincoln. 
Authors

Catherine Sargus-Patino, Elane C. Wright, Sarah A. Plautz, Jeremy R. Miles, Jeff L. Vallet, and Angela K. Pannier 


\title{
In vitro development of preimplantation porcine embryos using alginate hydrogels as a three-dimensional extracellular matrix
}

\author{
Catherine N. Sargus-Patino ${ }^{\mathrm{A}}$, Elane C. Wright ${ }^{\mathrm{B}}$, Sarah A. Plautz ${ }^{\mathrm{A}}$, \\ Jeremy R. Miles ${ }^{\mathrm{B}, \mathrm{D}}$, Jeff L. Vallet ${ }^{\mathrm{B}}$ and Angela K. Pannier ${ }^{\mathrm{A}, \mathrm{C}, \mathrm{D}}$ \\ A Department of Biological Systems Engineering, University of Nebraska-Lincoln, \\ PO Box 830726, Lincoln, NE 68583, USA. \\ ${ }^{B}$ USDA ${ }^{1}$-ARS US Meat Animal Research Center (USMARC), PO Box 166, \\ Clay Center, NE 68933, USA. \\ ${ }^{\mathrm{C}}$ Center for Nanohybrid Functional Materials, 220N Scott Engineering Center, \\ Lincoln, NE 68588, USA. \\ DCorresponding authors. Emails: jeremy.miles@ars.usda.gov; apannier2@unl.edu
}

\begin{abstract}
Between Days 10 and 12 of gestation, porcine embryos undergo a dramatic morphological change, known as elongation, with a corresponding increase in oestrogen production that triggers maternal recognition of pregnancy. Elongation deficiencies contribute to embryonic loss, but exact mechanisms of elongation are poorly understood due to the lack of an effective in vitro culture system. Our objective was to use alginate hydrogels as three-dimensional scaffolds that can mechanically support the in vitro development of preimplantation porcine embryos. White cross-bred gilts were bred at oestrus (Day 0) to Duroc boars and embryos were recovered on Days 9, 10 or 11 of gestation. Spherical embryos were randomly assigned to be encapsulated within double-layered $0.7 \%$ alginate beads or remain as non-encapsulated controls (ENC and CONT treatment groups, respectively) and were cultured for $96 \mathrm{~h}$. Every $24 \mathrm{~h}$, half the medium was replaced with fresh medium and an image of each embryo was recorded. At the termination of culture, embryo images were used to assess morphological changes and cell survival. 17ß-Oestradiol levels were measured in the removed media by radioimmunoassay. Real-time polymerase chain reaction was used to analyse steroidogenic transcript expression at $96 \mathrm{~h}$ in ENC and CONT embryos, as well as in vivo-developed control embryos (i.e. spherical, ovoid and tubular). Although no differences in cell survival were observed, 32\% $(P<0.001)$ of the surviving ENC embryos underwent morphological changes characterised by tubal formation with subsequent flattening, whereas none of the CONT embryos exhibited morphological changes. Expression of steroidogenic transcripts STAR, CYP11A1 and CYP19A1 was greater $(P<0.07)$ in ENC embryos with morphological changes $(\mathrm{ENC}+)$ compared with CONT embryos and ENC embryos with no morphological changes (ENC-), and was more similar to expression of later-stage in vivo-developed controls. Furthermore, a time-dependent increase $(P<0.001)$ in $17 \beta$-oestradiol was observed in culture media from ENC + compared with ENC - and CONT embryos. These results illustrate that preimplantation pig embryos encapsulated in alginate hydrogels can undergo morphological changes with increased expression of steroidogenic transcripts and oestrogen production, consistent with in vivo-developed embryos. This alginate culture system can serve as a tool for evaluating specific mechanisms of embryo elongation that could be targeted to improve pregnancy outcomes.
\end{abstract}

Additional keywords: elongation, pig, steroidogenesis.

Received 14 January 2013, accepted 13 June 2013, published online 6 August 2013

\section{Introduction}

In the pig, the preimplantation period of pregnancy is characterised by several developmental hallmarks. Between Days 10 and 12 of gestation, the porcine embryo undergoes a process known as elongation, transforming from an approximately 1-mm spherical structure to a long, thin filament that is $>100 \mathrm{~mm}$ in length (Geisert et al. 1982a; Pusateri et al. 1990). Unlike elongation in other domestic species, rapid elongation of the pig embryo occurs via cellular remodelling and differentiation of the trophectoderm rather than cellular hyperplasia

\footnotetext{
${ }^{1}$ USDA is an equal opportunity provider and employer.
} 
(Geisert et al. 1982a; Pusateri et al. 1990). During elongation, the embryo produces and secretes oestrogen, which serves as a key molecular signal for maternal recognition of pregnancy and modulates the production of proteins and growth factors within the uterus (Geisert et al. 1982b). Adequate elongation and successful implantation of the porcine embryo subsequently affect embryo spacing in the uterus, placental development and fetal growth, which have implications on uterine capacity, litter size and postnatal piglet health (Bennett and Leymaster 1989; Geisert and Schmitt 2002).

Approximately $20 \%$ of embryonic loss occurs during the preimplantation period of pregnancy in the pig, with a significant proportion of these losses occurring due to deficiencies in embryo elongation (Pope 1994). Early embryonic loss is poorly understood because exact physiological mechanisms by which the embryo elongates, and how these mechanisms are altered during embryonic loss, are not clear. Developing details of the mechanisms that regulate embryo elongation can allow for identification of specific biophysical, biomechanical and molecular factors that could serve as targets for improving pregnancy outcomes and neonatal piglet survival. An effective in vitro culture system that supports pig embryo elongation could be used to evaluate specific mechanisms of elongation. To date, attempts to initiate elongation in porcine embryos in vitro have been unsuccessful compared with other livestock species (Vejlsted et al. 2006). In cattle, limited elongation of embryos in vitro via physical induction was demonstrated using an agarose gel tube system (Brandão et al. 2004; Vajta et al. 2004; Machado et al. 2012). However, embryos cultured in this system had deficiencies in the development of the embryonic disk (Brandão et al. 2004; Vejlsted et al. 2006), which may have been the result of forced growth of the embryos to fill the tubes. Nevertheless, attempts to repeat these results using porcine embryos have been unsuccessful (Vejlsted et al. 2006).

Alginate is a commonly used biomaterial that is often used as an artificial extracellular matrix in tissue engineering applications (Lee and Mooney 2001). Alginate is a linear polysaccharide derived from brown algae composed of repeating units of $\beta$-D-mannuronic acid and $\alpha$-L-guluronic acid (Amsden and Turner 1999). One of the favourable properties of alginate as a biomaterial is its ability to form a hydrogel by ionic crosslinking of the guluronic residues in the presence of a divalent cation (Gombotz and Wee 1998). The alginate gel forms a meshlike structure that permits diffusion of nutrients and hormones essential for cell and tissue growth and development (Amsden and Turner 1999). Furthermore, alginate promotes negligible non-specific protein adsorption and cell adhesion, making it a suitable material for solely examining mechanical influence on tissue morphogenesis (Rowley et al. 1999; Miyajima et al. 2011). We hypothesise that previous failure of porcine embryos to elongate in vitro is due, at least in part, to inadequate culture systems lacking a three-dimensional (3D) structure for proper biomechanical support of embryo elongation. Traditional twodimensional (2D) cultures result in a disruption of cell-to-cell interactions as cells attach to the substrate and migrate away from the tissue (Smitz and Cortvrindt 2002; Kreeger et al. 2006). Alternatively, 3D culture systems can maintain embryo architecture and allow for direct physical interaction with the surrounding environment, which better mimics in vivo development. Therefore, our objective was to use alginate hydrogels to establish an in vitro culture system based on tissue engineering principles (i.e. 3D scaffolds) that can mechanically support the culture of preimplantation porcine embryos, ensure proper internal biomechanics and initiate morphological changes. In the present study, in vitro development was evaluated by characterising cellular survival, morphological changes, gene expression of steroidogenic and immunological transcripts, and $17 \beta$-oestradiol (E2) production from porcine embryos encapsulated (ENC) in alginate hydrogels or remaining as non-encapsulated controls (CONT).

\section{Materials and methods}

\section{Production and collection of embryos}

All animal protocols were approved by the US Meat Animal Research Center (USMARC) Animal Care and Use Committee and met the US Department of Agriculture (USDA) guidelines (USDA 1995) for the care and use of animals. Fifteen normally cycling White cross-bred gilts consisting of Landrace, Yorkshire and Duroc genetics were checked daily for oestrus. Following the first detection of oestrus (designated as Day 0), gilts were artificially inseminated with semen from a single Duroc sire with three sires represented across the replicate collections (International Boar Semen, Eldora, IA, USA; mention of trade names is necessary to report factually on available data; however, the USDA neither guarantees nor warrants the standard of the product, and the same by USDA implies no approval of the product to the exclusion of others that may also be suitable) and again $24 \mathrm{~h}$ later with the same sire. In three independent replicate collections, five gilts were killed at the USMARC abattoir on Day 9, 10 or 11 of gestation. After the gilts had been harvested, their reproductive tracts were immediately removed and each uterine horn was flushed with $20 \mathrm{~mL}\left(\sim 37^{\circ} \mathrm{C}\right)$ of HEPES-buffered RPMI-1640 medium (Invitrogen, Carlsbad, CA, USA) containing $1 \times$ antibiotics and antimycotics (Sigma-Aldrich, St Louis, MO, USA). Embryos were recovered and classified according to size and morphology using a standard stereomicroscope. Embryos were then washed with HEPES-buffered RPMI-1640 medium containing $1 \times$ antibiotics and antimycotics and $10 \%$ heat-inactivated fetal bovine serum (FBS; Invitrogen). From homogeneous litters, spherical embryos ( $\sim 1 \mathrm{~mm}$ diameter; range $0.5-1.5 \mathrm{~mm})$ recovered from gilts on Day 9 of gestation $(n=3$ gilts per replicate) were randomly assigned to the in vitro culture treatments (ENC or CONT); a subset of these embryos was collected and snap-frozen individually in liquid nitrogen to serve as initial in vivo-produced spherical control embryos for transcript expression. From homogeneous litters, ovoid $(5-10 \mathrm{~mm}$ diameter $)$ or tubular $(>10 \mathrm{~mm}$ diameter; range $11-20 \mathrm{~mm}$ ) embryos were recovered from the remaining gilts on Day 10 or 11 of gestation, respectively ( $n=1$ gilt per day per replicate) and snap-frozen individually in liquid nitrogen to serve as later-stage in vivo-developed control embryos for transcript expression. Uterine flushings from all pregnant gilts were collected, centrifuged at $2100 \mathrm{~g}$ for $20 \mathrm{~min}$ at room temperature to remove cellular debris and stored at $-20^{\circ} \mathrm{C}$ for later E2 and protein analysis. 


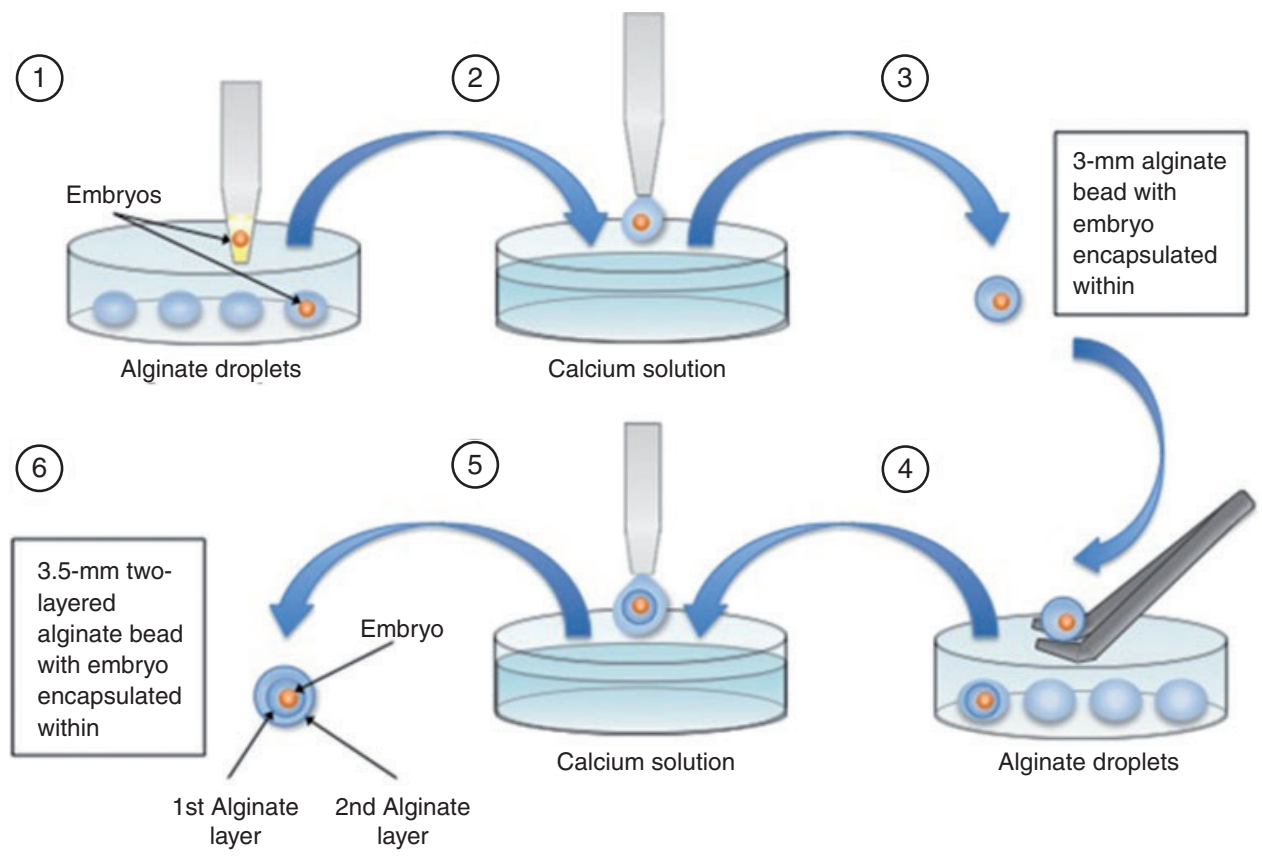

Fig. 1. Schematic illustration of the double-encapsulation method. (1) The embryo is transferred into the alginate droplet; (2) the droplet is pipetted into calcium solution; (3) an alginate bead forms around the embryo; (4) the alginate bead is transferred to a larger droplet of alginate; (5) the droplet with bead is pipetted into calcium solution; and (6) the second layer of alginate forms around the embryo. Following encapsulation, all embryos are washed and cultured individually in a single well within four-well NUNC plates (Thermo-Scientific, Rochester, NY, USA).

\section{Embryo encapsulation and culture}

Embryos assigned to the ENC group were encapsulated within alginate hydrogels using a double-encapsulation method (Fig. 1). Briefly, sodium alginate (Pronova UP MVG (>60\% guluronic acid; $\left.200000-300000 \mathrm{~g} \mathrm{~mol}^{-1}\right)$; NovaMatrix, Sandvika, Norway) was dissolved in sterile MilliQ water to a final concentration of $0.7 \%(\mathrm{w} / \mathrm{v})$. Droplets of warmed, sterile alginate solution were placed onto polystyrene culture dishes. A single embryo was pipetted into each droplet of alginate in a minimal amount of medium as not to dilute the alginate solution. Each droplet was then pipetted into a warmed, sterile cross-linking solution $(50 \mathrm{mM} \mathrm{CaCl}, 140 \mathrm{mM} \mathrm{NaCl})$ and allowed to gel for $3 \mathrm{~min}$. The resulting alginate beads with encapsulated embryos were then rinsed twice in HEPESbuffered RPMI-1640 medium containing $1 \times$ antibiotics and antimycotics and $10 \%$ FBS. Following rinsing, each bead was encapsulated in a second layer of alginate, using identical methods as described above for the initial encapsulation, to ensure complete encapsulation of each embryo. The final resulting beads were then washed once with $5 \% \mathrm{CO}_{2}$-gassed culture medium containing RPMI-1640, $1 \times$ antibiotics and antimycotics and 10\% FBS. The ENC and CONT embryos were cultured in individual wells in four-well NUNC plates (ThermoScientific, Rochester, NY, USA) with $1 \mathrm{~mL}$ culture medium for $96 \mathrm{~h}$ at $38.5^{\circ} \mathrm{C}$ and $5 \% \mathrm{CO}_{2}$ in air with $100 \%$ humidity.

\section{Characterisation of embryo viability and morphology}

Every $24 \mathrm{~h}$, a photographic image was recorded of each embryo using an SMZ1500 stereomicroscope (Nikon Instruments,
Melville, NY, USA). Based on visual appraisal of these images, embryos were classified into one of three in vitro treatment groups: CONT (non-encapsulated control embryos), ENC(encapsulated embryos with no observable morphological change) or ENC+ (encapsulated embryos with observable morphological changes). Embryos undergoing morphological changes were characterised by a tubular formation of the embryo within the gel and subsequent flattening of this tube, with many of these embryos migrating out of the gel forming a secondary spherical structure once freely in the culture medium (Fig. 2c). At the termination of culture, embryo survival was determined by assessing blastocyst fragmentation. Surviving embryos were characterised with no apparent sign of cellular degeneration, whereas dying embryos had significant cellular degeneration resulting in blastocyst compaction and darkening of the embryo. Embryo survival and death were confirmed in a subset $(n=10)$ of embryos using a fluorescein-ethidium bromide live-dead staining protocol, as reported previously (Edwards and Hansen 1996). Live or dead staining (green or red, respectively) was examined and imaged using an Axioplan2 fluorescence microscope (Zeiss, New York, NY, USA).

\section{Transcript expression analysis}

At the termination of the 96-h culture period, all embryos were imaged before being snap-frozen in liquid nitrogen. The CONT embryos were frozen in a small volume of culture medium $(\sim 5 \mu \mathrm{L})$, whereas ENC embryos were frozen within the alginate hydrogels. Preliminary analysis of RNA quality following extraction demonstrated no difference when embryos remained 
$\mathrm{Oh}$
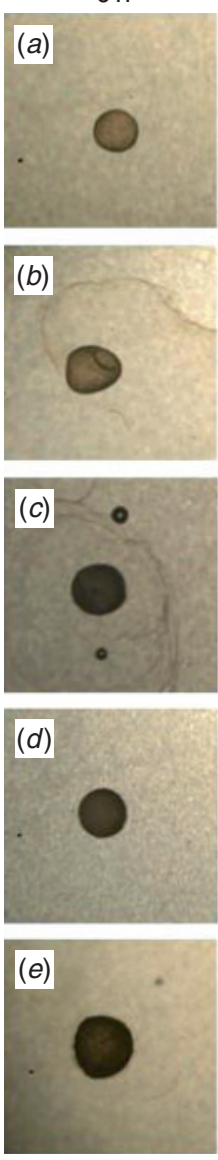

$24 \mathrm{~h}$
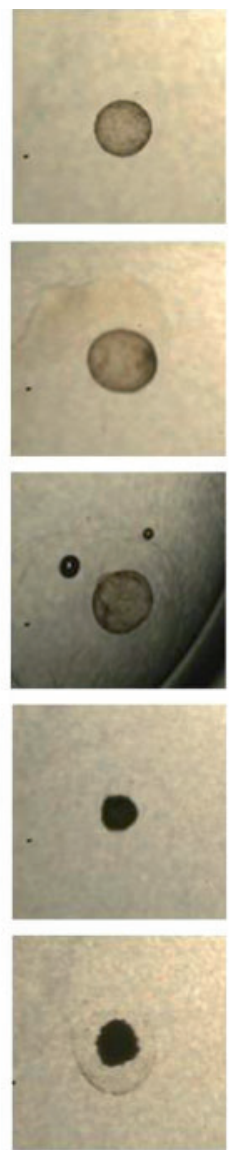

$48 \mathrm{~h}$
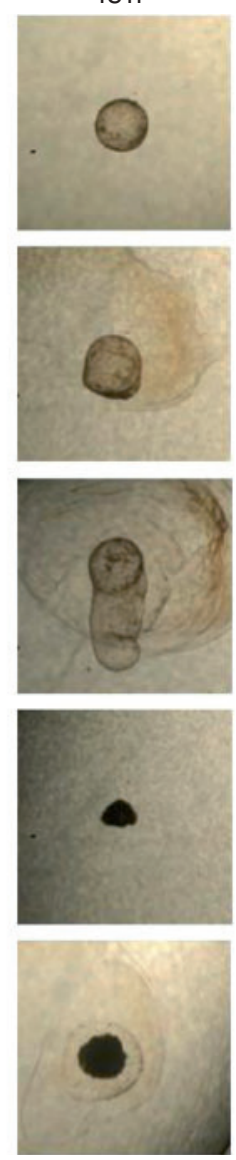

$72 \mathrm{~h}$
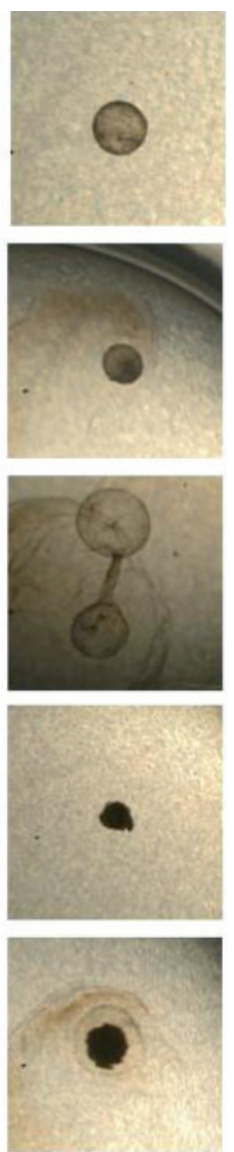

$96 \mathrm{~h}$
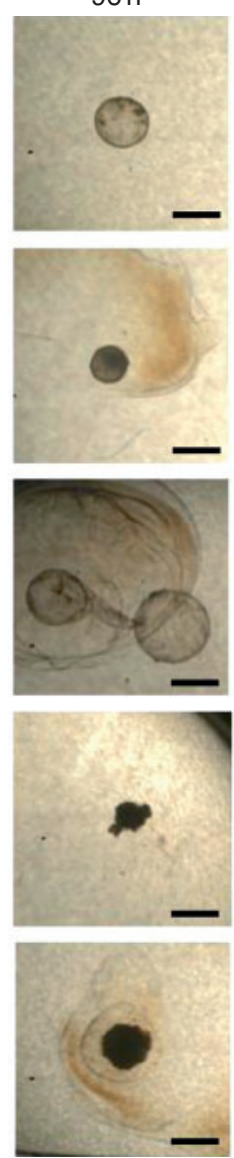

Live
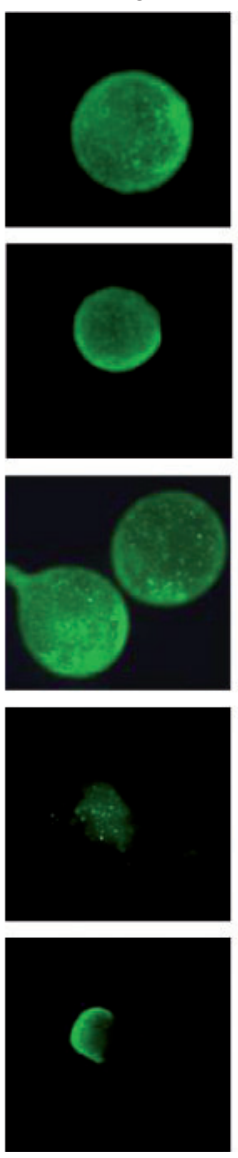

Dead
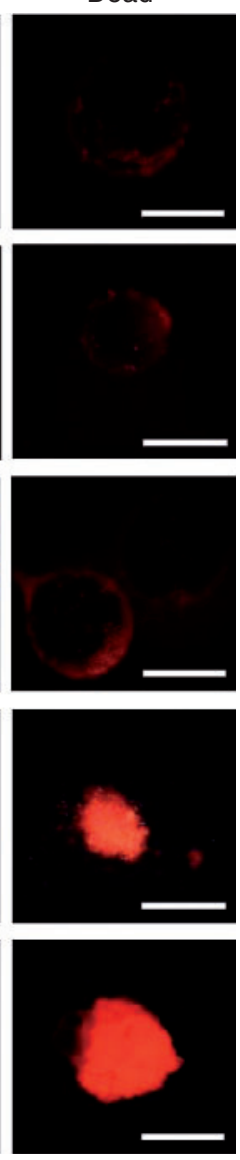

Fig. 2. Representative illustrations of morphological changes observed over $96 \mathrm{~h}$ of culture in embryos double-encapsulated in $0.7 \%$ alginate hydrogels or remaining as non-encapsulated control embryos, with corresponding cellular survival and death after $96 \mathrm{~h}$ culture. Surviving embryos that remained intact with no signs of cellular degeneration were classified into one of three in vitro treatment groups: (a) non-encapsulated control embryo with no observable morphological change (CONT); $(b)$ encapsulated embryo with no observable morphological change (ENC-); or $(c)$ encapsulated embryo with observable morphological changes $(\mathrm{ENC}+)$. Also shown are images of $(d)$ CONT and $(e)$ ENC- embryos that underwent significant cellular death, characterised by significant cellular degeneration resulting in blastocyst compaction and cellular darkening. The same representative embryo from each treatment group is shown across the different time points of culture and the live-dead staining. Scale bars $=1 \mathrm{~mm}$. Note the morphological changes of the ENC + embryo characterised by tubular formation of the embryo at $48 \mathrm{~h}$ that flattens by $72 \mathrm{~h}$, resulting in the embryo migrating out of the hydrogel by $96 \mathrm{~h}$. There were no observable differences in cellular survival (green stain) or death (red stain) between surviving embryos in the three treatment groups $(a-c)$; however, a greater proportion of dead cells (red stain) was observed in embryos classified as undergoing cellular death $(d, e)$.

encapsulated in the hydrogel versus de-encapsulating the embryos before collection and extraction (data not shown). Whole-cell (wc) RNA was extracted from individual embryos that survived (i.e. remaining intact with no cellular degeneration observed) throughout the duration of culture using the RNeasy Microkit (Qiagen, Valencia, CA, USA) with on-column DNase I treatment as described by the manufacturer. At the same time, wcRNA was extracted from in vivo-developed control embryos (i.e. spherical, ovoid and tubular) that had previously been collected. Quantification of wcRNA was performed with RiboGreen (Molecular Probes, Eugene, OR, USA) as per the manufacturer's instructions.

Using porcine-specific primers previously validated to amplify mRNA specific for the long-form variant of steroidogenic acute regulatory protein $(S T A R)$, cytochrome P450 side chain cleavage (CYP11A1), aromatase (CYP19A1) and interleukin-1 $\beta$ (IL1B; Blomberg and Zuelke 2005; Miles et al. 2008), transcript expression levels were assessed using a two-step, real-time quantitative polymerase chain reaction (qPCR) method with a Chromo4 real-time PCR detection system (Bio-Rad, Hercules, CA, USA). Briefly, reverse transcription was performed with $1 \mathrm{ng}$ wcRNA from the in vivodeveloped control (i.e. spherical, $n=7$; ovoid, $n=11$; tubular, $n=6)$, CONT $(n=7), \mathrm{ENC}-(n=13)$, and ENC $+(n=9)$ embryos using the iScript cDNA Synthesis kit (Bio-Rad) according to the manufacturer's instructions. In addition, a pooled sample of wcRNA consisting of $1 \mathrm{ng}$ wcRNA from all embryo treatment groups represented was reversed transcribed 
to generate a pooled cDNA sample that was used to generate a relative standard curve of cDNA for determining interassay $\mathrm{CV}$ and the relative quantity (RQ) of transcript expression. Each qPCR was assayed in duplicate and consisted of 50-pg equivalents of cDNA, $0.25 \mu \mathrm{M}$ of the appropriate forward and reverse primers and $12.5 \mu \mathrm{L}$ of $1 \times$ iTaq SYBR Green Supermix with ROX (Bio-Rad) in a $25 \mu \mathrm{L}$ reaction. All PCR conditions included denaturation $\left(95^{\circ} \mathrm{C}\right.$ for $\left.2 \mathrm{~min}\right)$ followed by amplification $\left(95^{\circ} \mathrm{C}\right.$ for $15 \mathrm{~s}, 60^{\circ} \mathrm{C}$ for $15 \mathrm{~s}$ and $70^{\circ} \mathrm{C}$ for $\left.45 \mathrm{~s}\right)$ for 40 cycles. Melting curve analysis and gel electrophoresis were used to confirm amplification of a single product of the predicted size.

Expression levels for each transcript were based on the threshold cycle $\left(\mathrm{C}_{\mathrm{T}}\right)$ values determined using Opticon Monitor 3 software (Bio-Rad). For each transcript, two assays were performed containing equal representation of all embryo treatment groups and a standard serial dilution of pooled cDNA consisting of 200-, 50-, 12.5-, 3.12- and 0.78-pg equivalents of cDNA. After converting the exponential $\mathrm{C}_{\mathrm{T}}$ to the linear $\mathrm{C}_{\mathrm{T}}$ using the formula $2^{-\mathrm{C}_{\mathrm{T}}}$ (Livak and Schmittgen 2001), the interassay $\mathrm{CV}$ for each transcript was determined using the pooled cDNA samples from each assay and the intraassay $\mathrm{CV}$ for each transcript was an average of all samples from both assays. The inter- and intraassay CV were $19.3 \%$ and $18.8 \%$, respectively, for $S T A R, 16.1 \%$ and $18.0 \%$, respectively, for CYP11A1, $14.9 \%$ and $20.0 \%$, respectively, for CYP19A1 and $7.3 \%$ and $20.4 \%$, respectively, for $I L 1 B$. Calculations of RQ values were made using a relative standard curve method by plotting treatment $C_{T}$ values against the logarithmic values of standard amounts of pooled cDNA (Čikoš et al. 2007). The RQ values are expressed as arbitrary units.

\section{Assays for $\mathrm{E} 2$ and protein}

Every $24 \mathrm{~h}$, half the culture medium $(500 \mu \mathrm{L})$ in each well was collected, stored at $-20^{\circ} \mathrm{C}$ for hormone and protein analysis and replaced in culture with fresh pregassed culture medium $(500 \mu \mathrm{L})$. In addition, wells containing culture medium alone (MEDIA) were included in each replicate and processed similarly to wells containing embryos. Analyses of E2 and protein content in the culture medium were performed in samples obtained after 24, 48, 72 and $96 \mathrm{~h}$ from the MEDIA group $(n=8)$ and cultures containing embryos represented in the qPCR analysis (i.e. CONT, $n=7$; ENC,$- n=13$; ENC,$+ n=9$ ). In addition, independent $\mathrm{E} 2$ and protein analyses were performed on uterine flushings collected from pregnant gilts on Days 9 $(n=9), 10(n=3)$ and $11(n=3)$. Culture media and uterine flushings were measured for E2 using a radioimmunoassay procedure described previously and validated in swine (Redmer and Day 1981; Miles et al. 2008). Culture media were ether extracted and measured for E2 in duplicate in one assay with an intraassay $\mathrm{CV}$ of $12.1 \%$. Uterine flushings were ether extracted and measured for E2 in duplicate in one assay with an intraassay $\mathrm{CV}$ of $13.0 \%$. In addition, culture media and uterine flushings were measured for total protein content using a bicinchoninic acid protein assay (Pierce, Rockford, IL, USA). The protein content in the culture media was measured in duplicate in six assays with inter- and intraassay CVs of $9.3 \%$ and $7.7 \%$, respectively. Protein content from uterine flushings was measured in duplicate in one assay with an intraassay CV of $2.4 \%$.

\section{Statistical analysis}

Proportional data for embryo survival and morphological changes following culture were analysed using the Chi-squared test and are reported as the least-squares mean (LSM) \pm s.e.m. after calculation with GLIMMIX model procedures (Steel et al. 1997; SAS 2003). Transcript expression data and E2 and protein levels in the culture media and uterine flushings were analysed using MIXED model procedures and are reported as the $\mathrm{LSM} \pm$ s.e.m. (Steel et al. 1997; SAS 2003). When a significant $F$-statistic was generated, mean values were separated using Dunnett's multiple comparison test (Steel et al. 1997; SAS 2003). Mean values were considered significantly different at $P \leq 0.05$, with tendencies defined as $P$-values between 0.06 and 0.10 . Transcript expression data were categorised into six treatment groups corresponding to in vivo-developed spherical, ovoid and tubular embryos and in vitro-cultured CONT, ENCand $\mathrm{ENC}+$ embryos, and the model included the fixed effects of treatment and replicate and the random effect of gilt within treatment. Culture E2 and protein data were categorised into four treatment groups (i.e. MEDIA, CONT, ENC - and ENC +) and the model included the fixed effects of treatment, culture time, replicate and treatment $\times$ culture time interaction, and the random effect of gilt within treatment $\times$ culture time interaction. Uterine flushing E2 and protein data were analysed with the model including day of gestation, replicate and the random effect of sire within day of gestation. To remove skewness and normally distribute the data, all transcript expression and E2 data were log transformed before statistical analysis and then back transformed to report observed means.

\section{Results}

\section{Evaluation of embryo viability and morphology}

Representative morphological changes observed over $96 \mathrm{~h}$ of culture of CONT and ENC embryos and their corresponding cellular survival and death, as determined by live-dead staining after $96 \mathrm{~h}$ culture, are shown in Fig. 2. Assessment of blastocyst degeneration at the termination of culture indicated that there was no significant difference $(P=0.22)$ in survival between the CONT and ENC embryos (Table 1). Embryo survival and death were further confirmed by live-dead staining a subset of embryos, which demonstrated no significant differences in cellular survival or death between treatment groups. Embryos classified as undergoing cellular degeneration had a greater proportion of dead cells (red), whereas surviving embryos had a greater proportion of live cells (green; Fig. 2). Morphological changes were only observed in the ENC embryos (Fig. 2c), whereas all CONT embryos remained spherical throughout the culture period (Fig. 2a; Table 1). The percentage of embryos that underwent morphological changes differed significantly between CONT and ENC groups when analysing all cultured embryos $(P<0.05)$ and only those embryos surviving $(P<0.001$; Table 1). The morphological changes observed in the ENC embryos were characterised by a tubular formation of the embryo within the gel and subsequent flattening of this tube, with many of these embryos migrating out of the gel forming a secondary spherical structure once freely in the culture medium (Fig. 2c). Although $32 \%$ of the surviving ENC embryos 
underwent morphological changes, a significant proportion of ENC embryos remained spherical throughout culture (Fig. $2 b$ ). As a result, encapsulated embryos were further classified as encapsulated embryos with no morphological changes (ENC-) or encapsulated embryos with morphological changes (ENC+).

Table 1. Summary of embryo survival and morphological changes observed following $96 \mathrm{~h}$ culture of either non-encapsulated control porcine embryos (CONT) or porcine embryos double encapsulated (ENC) in $0.7 \%$ alginate hydrogels

Values are reported as the least-squares mean \pm s.e.m., as determined using GLIMMIX analysis for the main effect of treatment (i.e. control vs encapsulated embryos). Significance for the effect of treatment was determined using Chi-squared analysis

\begin{tabular}{lccc}
\hline & CONT & ENC & $P$-value \\
\hline $\begin{array}{lcc}\text { No. embryos } \\
\text { Embryo survival }^{\mathrm{A}}(\%)\end{array}$ & $24.3 \pm 9.6(8)$ & $47.8 \pm 5.9(34)$ & 0.22 \\
$\begin{array}{l}\text { No morphological change } \\
\text { from all embryos (\%) }\end{array}$ & 100 & $83.1 \pm 4.4$ & 0.03 \\
$\begin{array}{l}\text { Morphological change } \\
\text { from all embryos (\%) }\end{array}$ & 0 & $16.9 \pm 4.4$ & 0.03 \\
$\begin{array}{l}\text { Morphological change } \\
\text { from surviving embryos (\%) }\end{array}$ & 0 & $32.3 \pm 8.0$ & $<0.001$ \\
\hline
\end{tabular}

${ }^{\mathrm{A}}$ Embryo survival was determined as a lack of blastocyst degeneration. Numbers in parentheses indicate the number of surviving embryos in each treatment group.

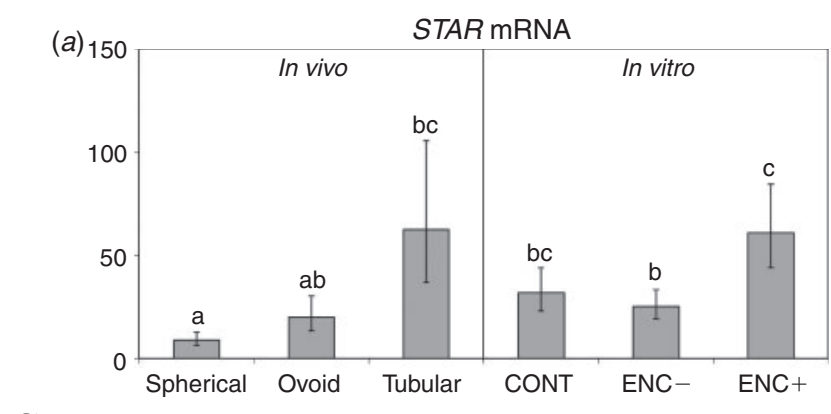

$\stackrel{\Upsilon}{\Upsilon}$

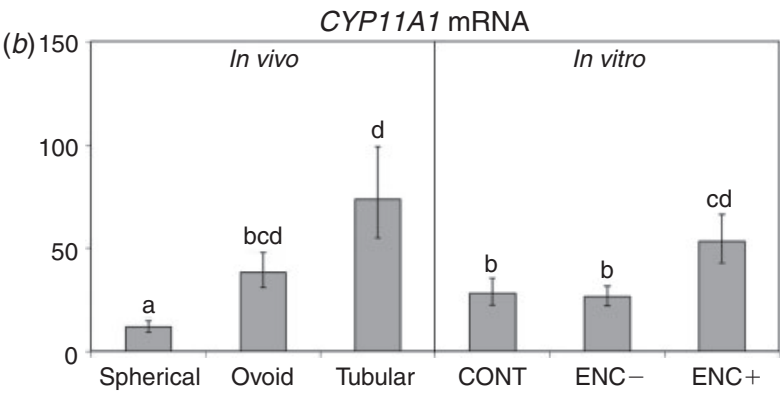

\section{Transcript expression levels}

The expression of STAR mRNA was increased $(P<0.05)$ in all in vitro treatment groups (CONT, $\mathrm{ENC}-$ and $\mathrm{ENC}+$ ) compared with the initial in vivo spherical control (Fig. $3 a$ ). However, STAR expression was greater $(P<0.05)$ in $\mathrm{ENC}+$ compared with ENC - embryos and tended to be greater $(P=0.07)$ in ENC+ versus CONT embryos (Fig. $3 a$ ). As a result, the expression pattern of STAR in CONT and ENC - embryos was more similar to that in in vivo ovoid embryos, whereas ENC+ embryos exhibited an expression pattern of STAR that was more similar to in vivo tubular embryos (Fig. $3 a$ ). Expression levels of CYP11A1 mRNA were increased $(P<0.05)$ in all in vitro treatment groups (CONT, ENC- and ENC + ) compared with their initial in vivo spherical controls (Fig. $3 b$ ). Furthermore, CYP11A1 expression was greater $(P<0.05)$ in $\mathrm{ENC}+$ compared with CONT and ENC - embryos. Again, as observed with STAR, the expression pattern of CYP11A1 in CONT and ENCembryos was more similar to that in in vivo ovoid embryos, whereas ENC + embryos exhibited an expression pattern of CYP11A1 that was more similar to in vivo tubular embryos (Fig. 3b). Expression of CYP19A1 mRNA was greater $(P<0.05)$ in ENC + than CONT and ENC - embryos (Fig. $3 c)$. In addition, CYP19A1 expression was significantly greater $(P<0.05)$ in $\mathrm{ENC}+$ embryos than in vivo spherical controls, whereas CYP19A1 expression did not differ between CONT, ENC - and in vivo spherical control embryos (Fig. 3c). Furthermore, CYP19A1 expression was decreased $(P<0.05)$ in
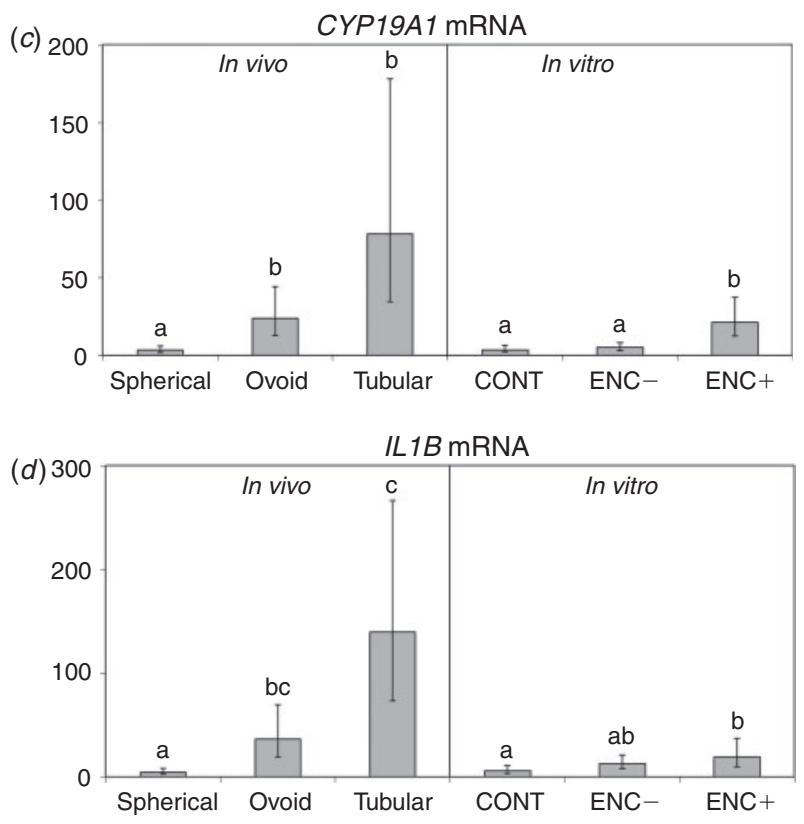

mbryo treatment

Fig. 3. Expression of $(a)$ steroidogenic acute regulatory protein $(S T A R),(b)$ cytochrome P450 side chain cleavage $(C Y P 11 A 1),(c)$ aromatase $(C Y P 19 A 1)$ and $(d)$ interleukin-1 $\beta(I L 1 B)$ mRNA, as determined by quantitative polymerase chain reaction, from in vivo-produced control embryos, in vitro-cultured non-encapsulated control embryos (CONT), encapsulated embryos with no observable morphological changes (ENC-) and encapsulated embryos with observable morphological changes (ENC + ). Data were log transformed before analysis and then back transformed to observable values. Data show relative quality (RQ) expressed as the least-squares mean \pm s.e.m. Statistical analysis demonstrated that embryo treatment had significant effects on the expression level of $(a) S T A R(P<0.01),(b) C Y P 11 A 1(P<0.001),(c) C Y P 19 A 1(P<0.01)$ and $(d) I L 1 B$ $(P<0.01)$. For each transcript, RQ values among treatment groups with different letters differ significantly $(P<0.05)$. 
CONT and ENC - embryos compared with in vivo ovoid and tubular embryos (Fig. 3c). In contrast, CYP19A1 expression did not differ between $\mathrm{ENC}+$ and the later-stage in vivo ovoid and tubular embryos, although the expression pattern was numerically more similar in $\mathrm{ENC}+$ and in vivo ovoid embryos (Fig. 3c). No significant differences were detected in the expression of ILIB mRNA between CONT, ENC - and in vivo spherical control embryos (Fig. $3 d$ ). However, $I L 1 B$ expression was increased in ENC+ compared with CONT and in vivo spherical control embryos (Fig. $3 d$ ). There was no significant difference in $I L 1 B$ expression between $\mathrm{ENC}+$ and in vivo ovoid embryos, but $I L 1 B$ expression was significantly $(P<0.05)$ decreased in $\mathrm{ENC}+$ compared with in vivo tubular embryos.

\section{Analysis of E2 and protein content}

A significant time-dependent increase $(P<0.001)$ in E2 levels in the culture media of encapsulated embryos (both ENC + and ENC-) was identified compared with culture media from

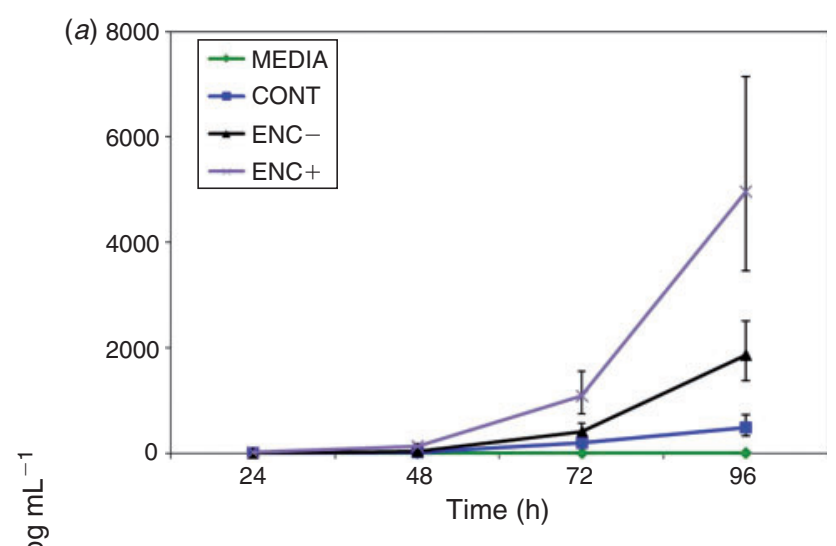

(b)

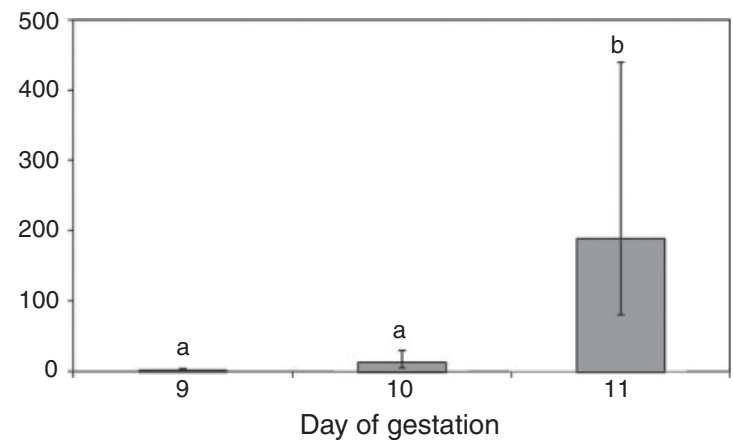

Fig. 4. (a) 17ß-Oestradiol (E2) concentrations in samples of the culture medium collected at $24,48,72$ and $96 \mathrm{~h}$ culture from wells containing medium only (MEDIA), non-encapsulated control embryos (CONT), encapsulated embryos with no observable morphological change (ENC-) or encapsulated embryos with observable morphological changes $(\mathrm{ENC}+)$. (b) E2 concentrations in uterine flushings from pregnant gilts on Days 9, 10 and 11 of gestation. Data were log transformed before analysis and then back transformed to observed values and are presented as the least-squares mean \pm s.e.m. Statistical analysis demonstrated a significant culture media treatment $\times$ culture time interaction $(P<0.001)$ for $\mathrm{E} 2$ concentration in which samples of culture medium from ENC + embryos had greater E2 concentrations than samples from the MEDIA, CONT and ENC - groups at 72 and $96 \mathrm{~h}$. Statistical analysis demonstrated a significant gestational day effect $(P<0.05)$ for $\mathrm{E} 2$ concentrations in uterine flushings.
CONT embryos and culture medium alone (Fig. 4a). Culture media from all embryo groups (i.e. CONT, ENC - and ENC+) had greater $(P<0.05)$ E2 levels than medium alone after $96 \mathrm{~h}$ of culture (Fig. 4a). Samples of culture medium from ENC+ embryos had significantly greater levels $(P=0.05)$ of E2 than samples from the CONT and ENC- groups after 72 and $96 \mathrm{~h}$ culture (Fig. $4 a$ ). In contrast, samples of culture medium from the ENC - group contained intermediate (but significantly different; $P<0.05$ ) levels of E2 between ENC + and CONT embryos after $96 \mathrm{~h}$ culture (Fig. $4 a$ ). A similar pattern of increased $(P<0.05)$ E2 production was observed in uterine flushings as pregnancy progressed from Day 9 to Days 10 and 11 of gestation, with the greatest E2 levels in uterine flushings on Day 11 of gestation (Fig. 4b).

There were no significant differences in total protein levels in samples of culture medium from the MEDIA, CONT, ENC- or

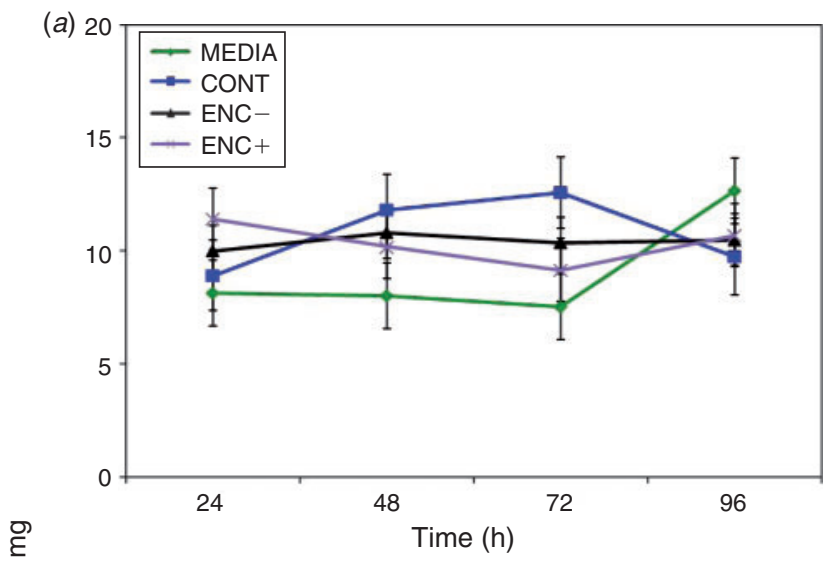

(b)

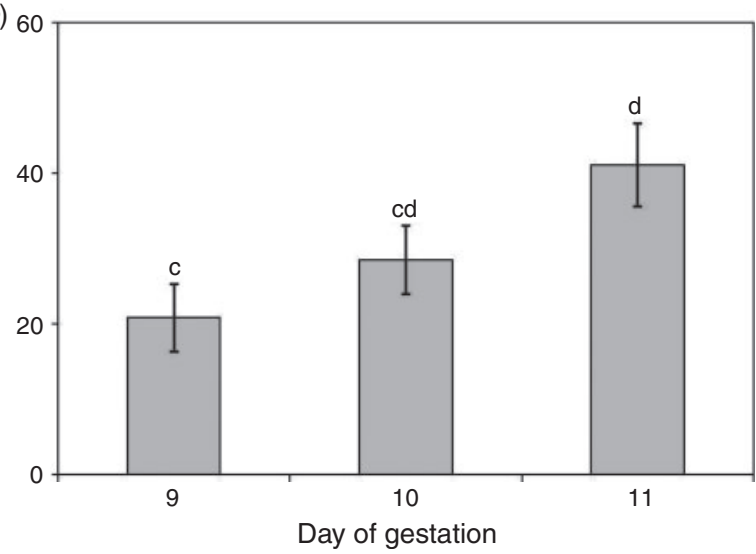

Fig. 5. (a) Total protein in samples of the culture medium collected at 24, 48, 72 and $96 \mathrm{~h}$ culture from wells containing medium only (MEDIA), nonencapsulated control embryos (CONT), encapsulated embryos with no observable morphological change (ENC-) and encapsulated embryos with observable morphological changes $(\mathrm{ENC}+)$. (b) Total protein in uterine flushings from pregnant gilts on Days 9, 10 and 11 of gestation. Data are presented as the least-squares mean \pm s.e.m. Statistical analysis demonstrated no significant $(P>0.10)$ effects for total protein collected from the culture media at $24,48,72$ and $96 \mathrm{~h}$ culture. Statistical analysis demonstrated a tendency $(P=0.09)$ for increased total protein in uterine flushings on Day 11 of gestation compared with Day 9, with Day 10 demonstrating an intermediate level of protein. 
ENC + groups (Fig. $5 a)$. In contrast, there tended $(P=0.09)$ to be a time-dependent increase in total protein levels from uterine flushings as pregnancy progressed from Day 9 to Day 11 of gestation (Fig. 5b).

\section{Discussion}

In the present study, alginate hydrogels were used as 3D matrices to support in vitro culture of porcine embryos during the preimplantation period. Previously, alginate hydrogels have been used to support in vitro development of early blastocysts in cattle (Yániz et al. 2002), as an artificial zona pellucida for mouse morulae (Krentz et al. 1993) and for the culture of ovarian follicles in mice (Kreeger et al. 2006; Xu et al. 2006a, 2006b; West et al. 2007) and non-human primates (Xu et al. 2009). However, the present study is the first to demonstrate the use of alginate hydrogels for porcine embryo culture. For those studies involving the encapsulation of early bovine embryos, $1.5 \%$ alginate hydrogels supported similar blastocyst rates as 2D controls; however, the alginate system resulted in decreased blastocyst hatching rates, suggesting that the percentage of alginate may have formed a matrix that was too rigid to allow for hatching (Yániz et al. 2002). Studies involving mouse ovarian follicle culture investigated mechanical properties of alginate gels of varying concentrations and demonstrated that decreasing alginate matrix stiffness enhanced follicle growth (West et al. 2007), linking biomechanical environmental factors with cell and/or tissue development and function. Specifically, these studies demonstrated that $0.7 \%$ alginate, the lowest concentration of alginate tested, formed a low-stiffness hydrogel that was the most permissive to mouse follicle growth, cellular differentiation and corresponding steroid production (West et al. 2007). Based on these findings, $0.7 \%$ alginate was used in the present study to provide sufficient mechanical support to properly maintain porcine embryo development, without inhibiting growth or survival.

Our in vitro culture system using alginate hydrogels was able to facilitate morphological changes of preimplantation porcine embryos by applying a tissue engineering approach to embryo culture. Although survival rates did not differ between the encapsulated and non-encapsulated embryos, morphological changes were only observed with embryos encapsulated in alginate hydrogels. The inability of the non-encapsulated control embryos to undergo morphological changes in vitro suggests that a $3 \mathrm{D}$ matrix is required to maintain appropriate embryo architecture for proper development during the elongation stage of gestation. These results support the findings of previous studies that demonstrated that both mechanical forces, generated from the stiffness of surrounding tissues or substrates, and cell-generated contractility regulate differentiation during embryonic development (Wozniak and Chen 2009). Our in vitro culture system using alginate hydrogel as a 3D matrix may be initiating embryo morphological change by stimulating biochemical signals within the embryo via mechanotransduction (Vogel and Sheetz 2006; Wozniak and Chen 2009) or by simply providing support for the 3D structure of the embryo to maintain its cell-to-cell communication, a feature that traditional culture systems have been lacking. The requirement of the $3 \mathrm{D}$ structure as a necessity to support morphological changes can be supported further by the observation that when ENC + embryos within our culture system exited the alginate hydrogel they formed a secondary spherical structure, likely due to fluid transport into the trophectoderm, which was observed in the non-encapsulated CONT embryos that did not have 3D support.

Although the percentage of total encapsulated embryos that exhibited morphological changes $(17 \%)$ may seem lower than ultimately desired, it is important to emphasise that if the encapsulated embryo survives within our newly developed in vitro system, it has a relatively high chance of initiating morphological changes $(32 \%)$ in simple basal medium conditions. In a previous study involving in vitro culture of bovine embryos in agarose gel tunnels, high levels of glucose in the culture medium may have resulted in rapid cell growth, contributing to the appearance of embryonic elongation (Brandão et al. 2004). Conversely, in the present study, no particular growth factors or nutrients were added to the culture medium, indicating that limited embryonic morphological changes can occur in vitro within alginate hydrogels without the addition of special media supplements. Although the percentage of porcine embryos initiating morphological changes in the present study is significantly less than reported in studies culturing bovine embryos in the agarose gel tunnel system, which ranged from $54 \%$ to $88 \%$ (Brandão et al. 2004; Vajta et al. 2004; Machado et al. 2012), it is important to highlight that these bovine studies with a high percentage of morphological changes were performed using embryos that were strictly produced in vitro and these embryos were selectively chosen as high-quality in vitroproduced embryos before being placed into the agarose gel tunnel system (Brandão et al. 2004; Vajta et al. 2004; Machado et al. 2012). In contrast, the present study only used in vivoproduced embryos collected on Day 9 of gestation in the pig. A previous study that investigated morphological changes using in vivo-produced bovine embryos within the agarose gel system reported a very low percentage $(3 \%)$ of in vivo-produced bovine embryos that initiated morphological changes following in vitro culture in the agarose gel system (Machado et al. 2013). This finding, in conjunction with the findings of the present study, illustrates that in vivo-produced embryos are not as efficient as in vitro-produced embryos at initiating morphological changes within in vitro culture systems, which is likely the result of the dramatic environmental change that in vivoproduced embryos experience going from the uterine environment to an in vitro environment. Therefore, the percentage of embryos that underwent morphological changes in the present study (i.e. $17 \%$ ) is relatively high for a study involving in vivoproduced embryos within an in vitro culture system, with only basal culture medium.

Several transcripts, such as steroidogenic transcripts (i.e. STAR, CYPY11A1 and CYP19A1) and immune response transcripts (i.e. $I L 1 B$ ), are increased in a similar pattern as oestrogen production during embryo elongation (Yelich et al. 1997; Blomberg et al. 2005). According to the results of the present study, STAR expression levels in ENC+ embryos were similar to those in tubular in vivo embryos, but greater than in ovoid in vivo embryos. In contrast STAR expression in CONT and $\mathrm{ENC}$ - embryos remained similar to that in ovoid in vivo embryos. STAR, which is a major initiator of steroidogenesis 
(Stocco and Clark 1997), has previously been found to be more abundantly expressed in elongated filamentous embryos than in ovoid embryos (Blomberg et al. 2005). Therefore, the STAR expression results of the present study suggest that embryos undergoing morphological changes within alginate hydrogels exhibit similar STAR expression trends as those that occur during initiation of elongation in vivo. Similar gene expression patterns were found for CYP11A1 and CYP19A1 within our in vitro culture system; these two are the additional transcripts encoding for rate-limiting proteins required for oestrogen synthesis (Blomberg et al. 2005). Expression of CYP11A1 and $C Y P 19 A 1$ was increased in ENC+ compared with CONT and ENC- embryos. These findings further suggest that encapsulated embryos that transformed morphologically during culture also changed at the level of gene expression, corresponding to the morphological transition and differential steroidogenic gene expression that occurs during the onset of elongation in vivo.

Proper interactions between the embryo and receptive uterine endometrium are also essential for supporting embryonic development and subsequent implantation (Ross et al. 2003). These interactions are initiated by the immune response cytokine $I L 1 B$, which is increased by the embryo during elongation and may be responsible for preventing conceptus rejection by suppressing the maternal immune response (Ross et al. 2003). Although our results demonstrate that the expression of $I L 1 B$ was greater in $\mathrm{ENC}+$ compared with CONT embryos, the expression of $I L 1 B$ was decreased in ENC + compared with tubular in vivo embryos. This outcome may suggest that direct interaction with the maternal endometrium is likely required for upregulation of $I L I B$ in the embryo, whereas upregulation of steroidogenic transcripts may be independent of maternalembryonic cross-talk.

In vivo oestrogen production by the porcine embryo increases during the elongation stage, stimulating the synthesis and release of numerous endometrial secretory proteins, which is essential for establishing maternal recognition of pregnancy (Geisert et al. 1982b; Yelich et al. 1997). Our results indicate that the ENC + embryos produce and secrete a greater amount of E2 at 72 and 96 h of culture compared with the CONT and ENCembryos. In addition, this upregulation of E2 in the culture medium of ENC+ embryos was independent of increased production of total protein within the culture medium. These results correspond with the increased expression of steroidogenic transcripts STAR, CYP11A1 and CYP19A1 in the ENC+ embryos, which supports previous findings of the direct correlation between these transcripts and steroid synthesis (Stocco and Clark 1997; Blomberg et al. 2005). Therefore, the E2 results further suggest that embryonic morphological changes induced by the alginate hydrogel culture system are indications of the initiation of elongation due to the corresponding increase in oestrogen production. The time-dependent increase in E2 observed in culture media of ENC + embryos followed a similar trend as observed for E2 levels in the uterine flushings as pregnancy advanced through this time period, with the greatest production of oestrogen observed on Day 11 of gestation when tubular embryos were present. Furthermore, protein increased in the uterine flushings as pregnancy advanced. Given that protein increases within the uterine milieu regardless of pregnancy status (Vallet et al. 1996), increased expression of steroidogenic transcripts and $\mathrm{E} 2$ production by ENC + embryos without a difference in protein levels within the culture medium further suggests that upregulation of steroidogenesis and subsequent oestrogen production may be independent of maternalembryonic cross-talk. It is important to highlight that there was a 48-h delay for embryos cultured within alginate hydrogels to initiate production and secretion of E2 into the culture medium, as well as for significant morphological changes to occur, which indicates a delay in development for in vitro-cultured compared with in vivo-developed embryos. A similar delay in morphological changes was observed in bovine embryos cultured in the agarose gel system compared with in vivo-developed embryos (Machado et al. 2013). This delay is likely due to the embryos recovering from the encapsulation technique and adjusting to the in vitro microenvironment.

Unlike elongation in other domestic animals, rapid elongation of the pig embryo has been primarily associated with cellular remodelling and differentiation rather than hyperplasia (Geisert and Yelich 1997). As a result, follow-up studies investigating potential markers for differentiation of the trophectoderm and mesoderm would be useful for measuring the level of differentiation from embryos undergoing morphological changes within our in vitro culture system. Furthermore, embryonic disc establishment, which was not monitored in the present study, will be included in follow-up studies to further evaluate the efficiency of our culture system. Monitoring the formation of the embryonic disc will be a useful technique for measuring the synchrony between trophoblast and embryonic disc development as we move forward to improve our in vitro culture system using alginate hydrogels.

In conclusion, the present study demonstrates an in vitro culture system that can support porcine embryo development during initiation of elongation. Our culture system, which uses alginate hydrogel as a 3D extracellular matrix for porcine embryo culture, has been shown to facilitate embryonic morphological changes with corresponding increases in steroidogenic transcript expression and oestrogen production, in a similar pattern as observed in in vivo-developed embryos. Our findings advocate the potential of our alginate culture system as an in vitro tool for evaluating and understanding specific mechanisms of embryo elongation. Further investigations with our 3D in vitro culture system can focus on the effects of certain growth factors, hormones, ligands and uterine epithelial cell coculture on the development of preimplantation porcine embryos. Specific factors regulating pig embryo elongation can be identified to develop strategies to improve pregnancy outcomes in the pig. Factors identified could then be manipulated through either direct intervention or marker-assisted selection of genomic markers to improve early embryonic survival, uterine capacity and preweaning piglet survival, which could have a significant impact on the profitability of swine production. Furthermore, our alginate culture system could be applied to embryos of other ungulate species, such as cattle and sheep, that also undergo embryo elongation during the preimplantation period of pregnancy to identify potential regulators of normal embryonic development in a variety of species. 


\section{Acknowledgements}

The authors thank Susan Hassler, Dave Sypherd, and Shanda Watts for technical assistance in collecting embryos, the USMARC swine crew for animal husbandry, the USMARC abattoir crew for assistance with killing the pigs and Drs. Jennifer Wood and Andrea Cupp for critical review of the manuscript. This work was supported by USDA-Agricultural Research Service, Current Research Information System Project No. 5438-31000084-00D and partially supported by the American Heart Association, the Nebraska Research Initiative, USDA Cooperative State Research, Education, and Extension Service-Nebraska (NEB-21-146), and the NebraskaInstitutional Development Award Program Networks of Biomedical Research Excellence program (National Institutes of Health P20 RR016469).

\section{References}

Amsden, B., and Turner, N. (1999). Diffusion characteristics of calcium alginate gels. Biotechnol. Bioeng. 65, 605-610. doi:10.1002/(SICI) 1097-0290(19991205)65:5<605::AID-BIT14>3.0.CO;2-C

Bennett, G. L., and Leymaster, K. A. (1989). Integration of ovulation rate, potential embryonic viability and uterine capacity into a model of litter size in swine. J. Anim. Sci. 67, 1230-1241.

Blomberg, L. A., and Zuelke, K. A. (2005). Expression analysis of the steroidogenic acute regulatory protein (STAR) gene in developing porcine conceptuses. Mol. Reprod. Dev. 72, 419-429. doi:10.1002/ MRD.20369

Blomberg, L. A., Long, E. L., Sonstegard, T. S., Van Tassell, C. P., Dobrinsky, J. R., and Zuelke, K. A. (2005). Serial analysis of gene expression during elongation of the peri-implantation porcine trophectoderm (conceptus). Physiol. Genomics 20, 188-194. doi:10.1152/PHYSIOLGENOMICS.00157.2004

Brandão, D. O., Maddox-Hyttel, P., Løvendahl, P., Rumpf, R., Stringfellow, D., and Callesen, H. (2004). Post hatching development: a novel system for extended in vitro culture of bovine embryos. Biol. Reprod. 71, 2048-2055. doi:10.1095/BIOLREPROD.103.025916

Čikoš, S., Bukovská, A., and Koppel, J. (2007). Relative quantification of mRNA: comparison of methods currently used for real-time PCR data analysis. BMC Mol. Biol. 8, 113-127. doi:10.1186/1471-2199-8-113

Edwards, J. L., and Hansen, P. J. (1996). Elevated temperature increases heat shock protein 70 synthesis in bovine two-cell embryos and compromises function of maturing oocytes. Biol. Reprod. 55, 341-346. doi:10.1095/ BIOLREPROD55.2.341

Geisert, R. D., and Schmitt, R. A. M. (2002). Early embryonic survival in the pig: can it be improved? J. Anim. Sci. 80(E. Suppl. 1), E54-E65.

Geisert, R. D., and Yelich, J. V. (1997). Regulation of conceptus development and attachment in pigs. J. Reprod. Fertil. Suppl. 52, 133-149.

Geisert, R. D., Brookbank, J. W., Roberts, R. M., and Bazer, F. W. (1982a). Establishment of pregnancy in the pig. 2. Cellular remodeling of the porcine blastocyst during elongation on Day-12 of pregnancy. Biol. Reprod. 27, 941-955. doi:10.1095/BIOLREPROD27.4.941

Geisert, R. D., Renegar, R. H., Thatcher, W. W., Roberts, R. M., and Bazer, F. W. (1982b). Establishment of pregnancy in the Pig. 1. Interrelationships between pre-implantation development of the pig blastocyst and uterine endometrial secretions. Biol. Reprod. 27, 925-939. doi:10.1095/ BIOLREPROD27.4.925

Gombotz, W. R., and Wee, S. (1998). Protein release from alginate matrices. Adv. Drug Deliv. Rev. 31, 267-285. doi:10.1016/S0169-409X(97) 00124-5

Kreeger, P. K., Deck, J. W., Woodruff, T. K., and Shea, L. D. (2006). The in vitro regulation of ovarian follicle development using alginateextracellular matrix gels. Biomaterials 27, 714-723. doi:10.1016/ J.BIOMATERIALS.2005.06.016
Krentz, K. J., Nebel, R. L., Canseco, R. S., and Mcgilliard, M. L. (1993). In vitro and in vivo development of mouse morulae encapsulated in 2-percent sodium alginate or 0.1-percent poly-L-lysine. Theriogenology 39, 655-667. doi:10.1016/0093-691X(93)90251-Y

Lee, K. Y., and Mooney, D. J. (2001). Hydrogels for tissue engineering. Chem. Rev. 101, 1869-1879. doi:10.1021/CR000108X

Livak, K. J., and Schmittgen, T. D. (2001). Analysis of relative gene expression data using real-time quantitative PCR and the 2(-Delta Delta C(T)) method. Methods 25, 402-408. doi:10.1006/METH.2001.1262

Machado, G. M., Caixeta, E. S., Lucci, C. M., Rumpf, R., Franco, M. M., and Dode, M. A. (2012). Post-hatching development of bovine embryos in vitro: the effects of tunnel preparation and gender. Zygote 20, 123-134. doi:10.1017/S0967199411000086

Machado, G. M., Ferreira, A. R., Guardieiro, M. M., Bastos, M. R., Carvalho, J. O., Lucci, C. M., Diesel, T. O., Sartori, R., Rumpf, R., Franco, M. M., and Dode, M. A. (2013). Morphology, sex ratio and gene expression of Day 14 in vivo and in vitro bovine embryos. Reprod. Fertil. Dev. 25, 600-608. doi:10.1071/RD11282

Miles, J. R., Freking, B. A., Blomberg, L. A., Vallet, J. L., and Zuelke, K. A. (2008). Conceptus development during blastocyst elongation in lines of pigs selected for increased uterine capacity or ovulation rate. J. Anim. Sci. 86, 2126-2134. doi:10.2527/JAS.2008-1066

Miyajima, H., Matsumoto, T., Sakai, T., Yamaguchi, S., An, S. H., Abe, M., Wakisaka, S., Lee, K. Y., Egusa, H., and Imazato, S. (2011). Hydrogel-based biomimetic environment for in vitro modulation of branching morphogenesis. Biomaterials 32, 6754-6763. doi:10.1016/ J.BIOMATERIALS.2011.05.072

Pope, W. F. (1994). Embryonic mortality in swine. In 'Embryonic Mortality in Domestic Species'. (Eds M. T. Zavy and R. D. Geisert.) pp. 53-77. (CRC Press: Boca Raton, FL.)

Pusateri, A. E., Rothschild, M. F., Warner, C. M., and Ford, S. P. (1990). Changes in morphology, cell number, cell-size and cellular estrogen content of individual littermate pig conceptuses on Day-9 to Day-13 of gestation. J. Anim. Sci. 68, 3727-3735.

Redmer, D. A., and Day, B. N. (1981). Ovarian activity and hormonal patterns in gilts fed allyl trenbolone. J. Anim. Sci. 53, 1088-1094.

Ross, J. W., Malayer, J. R., Ritchey, J. W., and Geisert, R. D. (2003). Characterization of the interleukin-1 beta system during porcine trophoblastic elongation and early placental attachment. Biol. Reprod. 69, 1251-1259. doi:10.1095/BIOLREPROD.103.015842

Rowley, J. A., Madlambayan, G., and Mooney, D. J. (1999). Alginate hydrogels as synthetic extracellular matrix materials. Biomaterials 20 , 45-53. doi:10.1016/S0142-9612(98)00107-0

SAS (2003). 'The SAS System for Windows, Release 9.1.' (Statistical Analysis System Institute: Cary, NC.)

Smitz, J. E., and Cortvrindt, R. G. (2002). The earliest stages of folliculogenesis in vitro. Reproduction 123, 185-202. doi:10.1530/ REP.0.1230185

Steel, R. G. D., Torrie, J. H., and Dickey, D. A. (1997). 'Principles and Procedures of Statistics: A Biometrical Approach', 3rd edn. (McGrawHill: New York.)

Stocco, D. M., and Clark, B. J. (1997). The role of the steroidogenic acute regulatory protein in steroidogenesis. Steroids 62, 29-36. doi:10.1016/ S0039-128X(96)00155-9

USDA (1995). Title 9 Code of Federal Regulations. Chapter 1, Subchapter AAnimal Welfare. (U.S. Government Printing Office: Washington, D.C.)

Vajta, G., Alexopoulos, N. I., and Callesen, H. (2004). Rapid growth and elongation of bovine blastocysts in vitro in a three-dimensional gel system. Theriogenology 62, 1253-1263. doi:10.1016/J.THERIOGEN OLOGY.2004.01.007

Vallet, J. L., Christenson, R. K., and McGuire, W. J. (1996). Association between uteroferrin, retinol-binding protein, and transferrin within the 
uterine and conceptus compartments during pregnancy in swine. Biol. Reprod. 55, 1172-1178. doi:10.1095/BIOLREPROD55.5.1172

Vejlsted, M., Du, Y., Vajta, G., and Maddox-Hyttel, P. (2006). Post-hatching development of the porcine and bovine embryo: defining criteria for expected development in vivo and in vitro. Theriogenology 65, 153-165. doi:10.1016/J.THERIOGENOLOGY.2005.09.021

Vogel, V., and Sheetz, M. (2006). Local force and geometry sensing regulate cell functions. Nat. Rev. Mol. Cell Biol. 7, 265-275. doi:10.1038 NRM1890

West, E. R., Xu, M., Woodruff, T. K., and Shea, L. D. (2007). Physical properties of alginate hydrogels and their effects on in vitro follicle development. Biomaterials 28, 4439-4448. doi:10.1016/J.BIOMATER IALS.2007.07.001

Wozniak, M. A., and Chen, C. S. (2009). Mechanotransduction in development: a growing role for contractility. Nat. Rev. Mol. Cell Biol. 10, 34-43. doi:10.1038/NRM2592

Xu, M., Kreeger, P. K., Shea, L. D., and Woodruff, T. K. (2006a). Tissueengineered follicles produce live, fertile offspring. Tissue Eng. 12, 2739-2746. doi:10.1089/TEN.2006.12.2739
Xu, M., West, E., Shea, L. D., and Woodruff, T. K. (2006b). Identification of a stage-specific permissive in vitro culture environment for follicle growth and oocyte development. Biol. Reprod. 75, 916-923. doi:10.1095/BIOLREPROD.106.054833

Xu, M., West-Farrell, E. R., Stouffer, R. L., Shea, L. D., Woodruff, T. K., and Zelinski, M. B. (2009). Encapsulated three-dimensional culture supports development of nonhuman primate secondary follicles. Biol. Reprod. 81, 587-594. doi:10.1095/BIOLREPROD.108.074732

Yániz, J. L., Santolaria, P., and López-Gatius, F. (2002). In vitro development of bovine embryos encapsulated in sodium alginate. J. Vet. Med. A Physiol. Pathol. Clin. Med. 49, 393-395. doi:10.1046/J.1439-0442. 2002.00463.X

Yelich, J. V., Pomp, D., and Geisert, R. D. (1997). Ontogeny of elongation and gene expression in the early developing porcine conceptus. Biol. Reprod. 57, 1256-1265. doi:10.1095/BIOLREPROD57.5.1256 